\title{
Aerodynamics and Performance Verifications of Test Methods for Laboratory Fume Cupboards
}

\section{LI-CHING TSENG ${ }^{1}$, RONG FUNG HUANG ${ }^{2} *$, CHIH-CHIEH CHEN $^{1}$ and CHENG-PING CHANG}

\author{
${ }^{1}$ Institute of Occupational Medicine and Industrial Hygiene, National Taiwan University, \\ 1 Jen-Ai Road, Section 1, Taipei; ${ }^{2}$ Department of Mechanical Engineering, National Taiwan \\ University of Science and Technology, 43 Keelung Road, Section 4, Taipei, ${ }^{3}$ Institute of Occupational \\ Safety and Health, Council of Labor Affairs, 99 Lane 407, Hengke Road, Sijhih City, Taipei, Taiwan
}

Received 23 December 2005; in final form 28 June 2006; Published online 19 August 2006

\begin{abstract}
The laser-light-sheet-assisted smoke flow visualization technique is performed on a full-size, transparent, commercial grade chemical fume cupboard to diagnose the flow characteristics and to verify the validity of several current containment test methods. The visualized flow patterns identify the recirculation areas that would inevitably exist in the conventional fume cupboards because of the fundamental configurations and structures. The large-scale vortex structures exist around the side walls, the doorsill of the cupboard and in the vicinity of the nearwake region of the manikin. The identified recirculation areas are taken as the 'dangerous' regions where the risk of turbulent dispersion of contaminants may be high. Several existing tracer gas containment test methods (BS 7258:1994, prEN 14175-3:2003 and ANSI/ASHRAE 110:1995) are conducted to verify the effectiveness of these methods in detecting the contaminant leakage. By comparing the results of the flow visualization and the tracer gas tests, it is found that the local recirculation regions are more prone to contaminant leakage because of the complex interaction between the shear layers and the smoke movement through the mechanism of turbulent dispersion. From the point of view of aerodynamics, the present study verifies that the methodology of the prEN 14175-3:2003 protocol can produce more reliable and consistent results because it is based on the region-by-region measurement and encompasses the most area of the entire recirculation zone of the cupboard. A modified test method combined with the region-by-region approach at the presence of the manikin shows substantially different results of the containment. A better performance test method which can describe an operator's exposure and the correlation between flow characteristics and the contaminant leakage properties is therefore suggested.
\end{abstract}

Keywords: flow visualization; laboratory fume cupboard; performance; tracer gas; turbulent dispersion; vortex

\section{INTRODUCTION}

One of the most important safety devices in a laboratory is the properly functioning fume cupboard. A laboratory fume cupboard is a boxlike structure enclosing a source of potential air contamination, with one open or partially open side. In the fume cupboard, hazardous chemicals released from experiments are drawn away from the worker and exhausted by fans. Laboratory fume cupboards and ventilation equipment are designed for the protection

\footnotetext{
*Author to whom correspondence should be addressed. Tel: +886 22737 6488; fax: +886 22737 6460;

e-mail: rfhuang@mail.ntust.edu.tw
}

of personnel by preventing contaminants such as vapors, dusts, mists and fumes from being released into the laboratory and building environment.

The performance of a fume cupboard is determined by a complex interaction of factors from the working chamber, the exhaust system, fume cupboard location, make-up air, system indicators and operational parameters. Fume cupboard performance evaluation strategies differ in method and complexity. The test protocols used to judge the performance of a fume cupboard may include face velocity test, flow visualization test or tracer gas test. Face velocity measurements determine the average velocity of air moving perpendicular to the hood face. This test examines the uniformity of face velocity across the face of 
the fume hood opening at different sash positions. A flow visualization test requires the generation of smoke streams at target locations within the cupboard. It provides a visual understanding of the air flow currents that exist within the cupboard.

Fletcher and Johnson (1992a, 1992b), Saunders (1993), Volin et al. (1998), Maupins and Hitchings (1998) and Ekberg and Melin (2000) suggest that maintaining a specific face velocity does not assure fume cupboard containment, because the correlation between the face velocity and the hood performance is not observed. Although face velocity is not a direct measure of a cupboard's containment to provide operator protection, regulatory and professional organizations require a variety of face velocities as design and operating criteria.

The quantitative containment tests can provide more valuable information of ventilation system performance than that provided by the traditional face velocity measurement. A similar system for surface tanks was suggested by Marzal et al. (2002, 2003a, 2003b). Usually, a tracer gas such as sulfur hexafluoride $\left(\mathrm{SF}_{6}\right)$ is delivered into the cupboard at a known rate and measurements of concentration are collected around the cupboard to determine gas escape. A number of national standards exist. The British Standards Institution (1994) developed BS 7258: Part 4: 1994 'Laboratory Fume Cupboards' in which the containment ability is tested on the basis of the average of grid-overall of the cupboard face. The strategy of the ANSI/ASHRAE 110-1995 'Method of Testing Performance of Laboratory Fume Hoods' developed by ASHARE (1995) attempts to measure the $\mathrm{SF}_{6}$ concentration in the breathing zone of a fume cupboard operator. The European Committee for Standardization (2003) proposed prEN 141753:2003 Fume Cupboards Part 3: Type Test Methods'. This test methodology incorporates an inner plane test (static test) and outer plane tests (static and dynamic sash tests) together with a robustness test. The inner plane measurement is proposed to determine the local grid $\mathrm{SF}_{6}$ concentration based on six locations of the sampling grids with respect to the sash opening of the fume cupboard. We propose in this paper a modification of the prEN 14175-3:2003 protocol based on the combination of region-by-region approach and the measurement of the near-wake of an operator. The characteristics of these test methods may differ in the configuration of tracer gas releasing, source injection and sampling grid arrangement.

Durst and Pereira (1991, 1992), Hu et al. (1996, 1998), Kirkpatrick and Reither (1998), Nicholson et al. (2000) and Lan and Viswanathan (2001) concentrate on using numerical analysis to predict flow patterns and the containment ability of fume cupboards. The key element in the flow phenomena of the fume cupboard is identified as the boundary layer turbulence, which contributes to contaminant leakage. The potential of various factors that can cause the leakage of contaminants from the fume hood are investigated, in particular the sash height, the effect of the location of the exhaust outlet, and how exterior obstructions of different shapes and sizes affect on the flow patterns. However, detailed experimental data on this subject and the extent of the understanding on the physical mechanisms governing the leakage of contaminant during the ventilation process are limited and therefore warrant further investigation.

The present study uses the laser-light-sheetassisted smoke flow visualization method and tracer gas measurement to diagnose the global/local flow structure and the tracer gas leakage of a chemical fume cupboard, respectively. The effects of the source position, geometric feature of the cupboard and the presence of an operator on the performance of the fume cupboard are investigated experimentally. The containment tests by employing the BS 7258:1994 standard, the prEN 14175:2003 protocol, the ANSI/ASHRAE 110-1995 standard and the modified method are compared to clarify the behavior of the flow structure and the containment ability.

\section{EXPERIMENTAL ARRANGEMENTS}

The experimental setup includes a laboratory fume cupboard model, an exhaust fan and instruments, as shown in Fig. 1. The test fume cupboard has an $850 \mathrm{~mm} \times 1200 \mathrm{~mm}$ aperture and is made of transparent acrylic plates so that the laser beams can pass through. The fume cupboard consists of a baffle across the rear wall. The baffle has a top slot and a bottom slot to help effectively remove the contaminants through the cupboard. The top panel of the cupboard has an exhaust collar to connect the exhaust duct to the cupboard. An AC motor/centrifugal fan provides the suction. The suction flow rate is measured by a homemade venturi flow meter along with a calibrated pressure gauge. The operating suction flow rate is set at $0.36 \mathrm{~m}^{3} \mathrm{~s}^{-1}$. The error of the suction flow rate measurement is $<1 \%$ of the reading. Measurement is conducted with the sash at a height of $500 \mathrm{~mm}$.

Experiments are carried out in a well-controlled test room. The test room is a typical laboratory room with dimensions of $19 \mathrm{~m} \times 16 \mathrm{~m} \times 5 \mathrm{~m}$. During the experiment, turbulence and external interference from external sources such as air supply diffusers, doors and traffic in the room are restricted.

The experimental study includes two parts: (i) flow visualization and (ii) tracer gas test.

\section{Flow visualization}

The experimental apparatus for flow visualization is shown in Fig. 2. A paraffin oil mist is produced in the smoke generator and continuously seeded through 

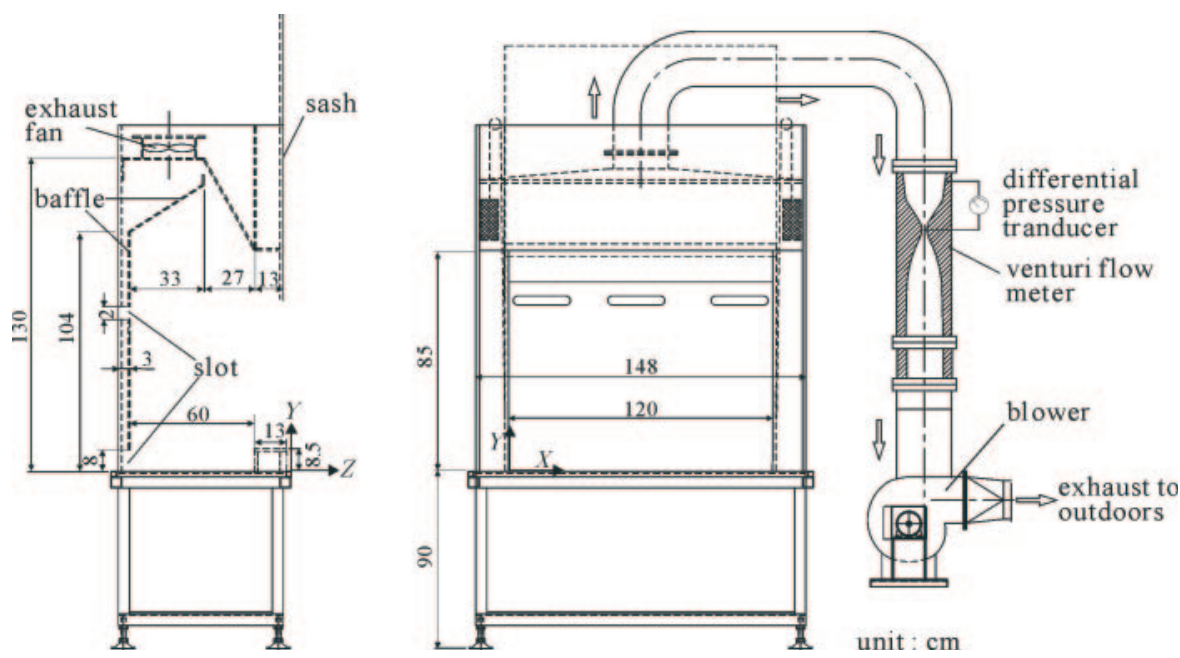

Fig. 1. Experimental setup.

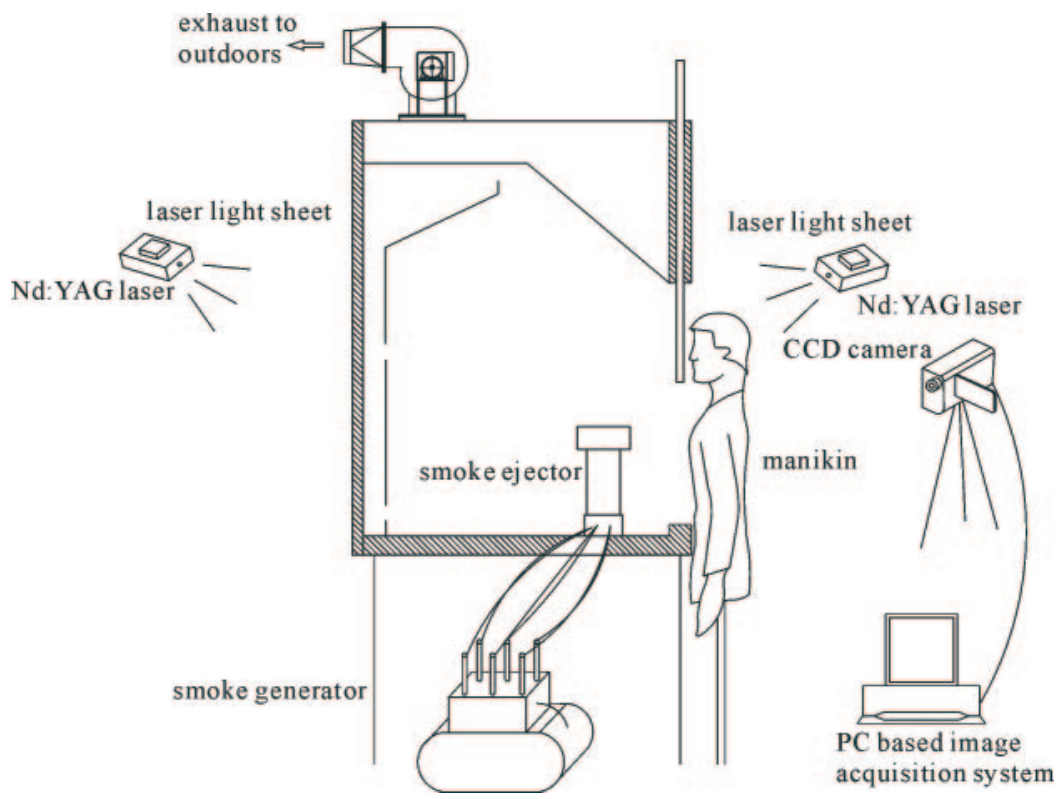

Fig. 2. Experimental apparatus for flow visualization.

the homemade smoke ejector into the test section. The diameter of the oil-mist particles, measured by a Malvern $2600 \mathrm{C}$ particle analyzer, is $1.7 \pm 0.2 \mu \mathrm{m}$. The density of this particle is $0.821 \mathrm{~g} \mathrm{ml}^{-1}$. Without considering the effect of turbulent diffusion, the relaxation time constant is estimated to be $<7.7 \times 10^{-5} \mathrm{~s}$ and the Stokes number is in the order of $10^{-6}$ within the range of experiment. Therefore, Flagan and Seinfeld (1988) suggest that the seeded particles can properly follow the flow fluctuations at least up to $10 \mathrm{kHz}$.

The streams of the visible smoke are discharged by two different means: (i) for simulating the contaminant transport process, the smoke is generated from the smoke generator and piped to the smoke ejector; (ii) for better visualization of the flow structure in the test section in corresponding to the contaminant transport behavior, the ejector tubes are positioned at targeted sections by releasing a uniform outlet velocity of smoke. The smoke generator used in this experiment is equipped with six tubes, and each tube is connected to a ball valve. The smoke is distributed through a wire mesh outlet diffuser. The laser beam from Nd:YAG laser is transmitted through an optical fiber and connected to a $20^{\circ}$ laser-light sheet expander. The laser-light sheet expander is mounted on an adjustable block so that the light sheet can be easily aligned on different planes. The laser-light sheet is adjusted to a thickness of $\sim 0.5 \mathrm{~mm}$. A Hi-8 CCD camera is used to record the particle images. The camera is equipped with an asynchronous 
variable electronic shutter, ranging from $1 / 12000$ to $1 / 60 \mathrm{~s}$ and could record images at $30 \mathrm{fps}$.

The mode on the presence of the manikin's related to the performance of the cupboard is important to assess. In order to study the effects of the source position and the manikin's presence on the flow structure, the visualization experiments are conducted in the following procedures:

Visualization of effect of source position. The arrangement of the smoke ejector configuration and the release rate of the smoke are in accordance with the BS 7258 Part 4:1994 standard. The release rate of the smoke is $2.41 \mathrm{~min}^{-1}$. The velocity of the smoke at outlet of the injector is $5.6 \mathrm{~cm} \mathrm{~s}^{-1}$. The smoke ejector is located $150 \mathrm{~mm}$ behind the sash plane with its axis parallel to the plane of the sash. The top of the ejector is either $150 \mathrm{~mm}$ above the work surface or $150 \mathrm{~mm}$ below the upper sash boundary at a point $150 \mathrm{~mm}$ from one of the side walls or at the horizontal centerline of the two sidewalls, i.e. the positions $\mathrm{S} 1, \mathrm{~S} 2$, S3, S4, S5 and S6, as shown in Fig. 3a. The smoke ejector is arranged successively at each individual position during the test.

Visualization of effect of manikin's presence. In order to observe the effect of the near-wake region of the manikin, experiments with a manikin standing in front of the cupboard are performed so that comparisons can be made with the situation without the manikin's presence.

The arrangement of the smoke ejector configuration and positions and the release rate of the smoke are in accordance with the ANSI/ASHRAE 110-1995 standard. The velocity of the smoke at the outlet of the injector is $0.59 \mathrm{~cm} \mathrm{~s}^{-1}$. When the manikin is present, its vertical centerline is in line with the vertical centerline of the smoke ejector. The nose tip of the manikin (the region of the breathing zone) is $660 \mathrm{~mm}$ above the work surface and $75 \mathrm{~mm}$ in front of the sash. The manikin is fully clothed in three-dimensions. The total height is $1630 \mathrm{~mm}$ and the shoulder height is $1380 \mathrm{~mm}$. The shoulder width is $450 \mathrm{~mm}$ and the nose length is $23 \mathrm{~mm}$. The arms of the manikin hang at its sides. The human body is a heat source and the convective currents at the body may act as vehicle for contaminant (Johnson and Fletcher, 1996). The factors with respect to both heat source at the human body and fume cupboard air system are not discussed in the study. For the present study, use of a non-heated manikin to replace a human body may not be able to fully represent the unique feature of the human body.

\section{Tracer gas test}

A pressure gauge, a needle value and a calibrated rotameter are used to control the flow rate. The mean and peak value of $\mathrm{SF}_{6}$ concentration are measured and recorded with the SapphIRe ${ }^{\mathrm{TM}}$ analyzer. According to the manufacturer, the lower and upper detection limits for $\mathrm{SF}_{6}$ using this instrument were 0.01 and $100 \mathrm{ppm}$, respectively. The sampling flow rate is $141 \mathrm{~min}^{-1}$. The accuracy of the instrument is $10 \%$ of the reading for concentrations between 0 and $1 \mathrm{ppm}, 20 \%$ of the reading between 1 and $4 \mathrm{ppm}$. The time constant of the instrument is $1 \mathrm{~s}$ and the sampling rate of the detection is 20 readings per second. Average value over 10 s (i.e. 200 readings) is recorded so that the recorded data rate is $0.1 \mathrm{~Hz}$.

The experimental procedures are conducted in accordance with the BS 7258 Part 4: 1994 standard, the prEN 14175-3:2003 protocol, the ANSI/ ASHRAE 110-1995 standard and the modified method as follows:

Static test following BS 7258 Part 4: 1994 standard. The tracer gas measurements are performed in accordance with the BS 7258 Part 4:1994 standard by using $10 \% \mathrm{SF}_{6}$ in nitrogen gas $\left(\mathrm{N}_{2}\right)$ as the tracer gas. The release rate of the tracer gas and the positions of the tracer gas ejector are arranged in the way described in the section of the flow visualization arrangement. The tracer gas ejector consists of a cylindrical filter funnel fitted with a sintered glass disc near its base. The glass disc is $30 \mathrm{~mm}$ in diameter and having a pore rating of $\mathrm{P} 40$ in accordance with this standard. The velocity of the gas at the outlet of the injector is $5.6 \mathrm{~cm} \mathrm{~s}^{-1}$. As shown in Fig. 3a, 15 sampling probes are positioned at the grids (the black dots) on the rectangular area in the plane of sash. The corners of the rectangle are $50 \mathrm{~mm}$ from the sides of the aperture. The sampling probes are connected with 15 equal length tubes so that 15 gas streams are homogeneously mixed in a homemade collector. The detector probe is affixed to the outlet of the collector. Tracer gas samples are taken through a stainless steel tube of $13 \mathrm{~mm}$ internal diameter, which is fitted with a diffuser of $30 \mathrm{~mm}$ internal diameter at inlet end of suction velocity $3.7 \mathrm{~cm} \mathrm{~s}^{-1}$. Sampling is taken for $600 \mathrm{~s}$ and the data for the initial period of $180 \mathrm{~s}$ are discarded.

Static test following prEN 14175-3:2003 protocol. The measurements are performed in accordance with the prEN 14175-3:2003 protocol by using $10 \% \mathrm{SF}_{6}$ in $\mathrm{N}_{2}$ as the tracer gas. The tracer gas ejector is a hollow cylinder made of sintered metal with a length $25 \mathrm{~mm}$ and a diameter $15 \mathrm{~mm}$ in accordance with this method. The release rate of the tracer gas is $2.01 \mathrm{~min}^{-1}$. The velocity of the gas at outlet of the injector is $18.86 \mathrm{~cm} \mathrm{~s}^{-1}$. As shown in Fig. 3b, nine sampling probes are arranged in a grid based on a square area of $200 \mathrm{~mm} \times 200 \mathrm{~mm}$. Each sampling probe has a funnel-shaped effuser of $3 \mathrm{~cm}$ inner diameter at the inlet. The suction velocity at the inlet of the funnel-shaped effuser is $\sim 4 \mathrm{~cm} \mathrm{~s}^{-1}$. The tracer gas ejector is held vertically facing up with its center in line with and $15 \mathrm{~cm}$ from the center of the sampling probe grid. The sampling probe grid, which is formed by the 
(a)

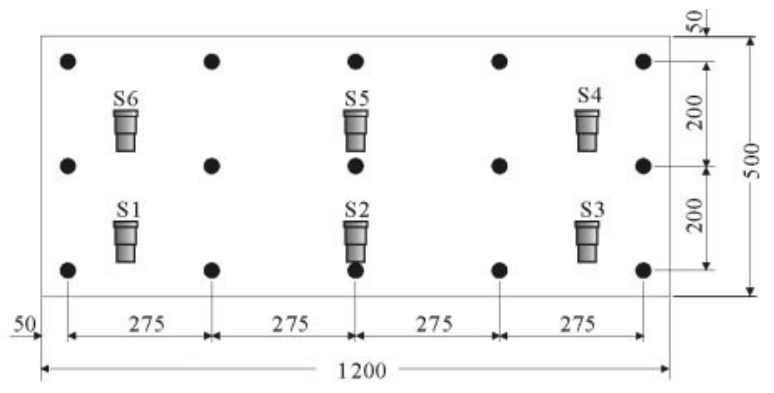

(b)

unit : mm

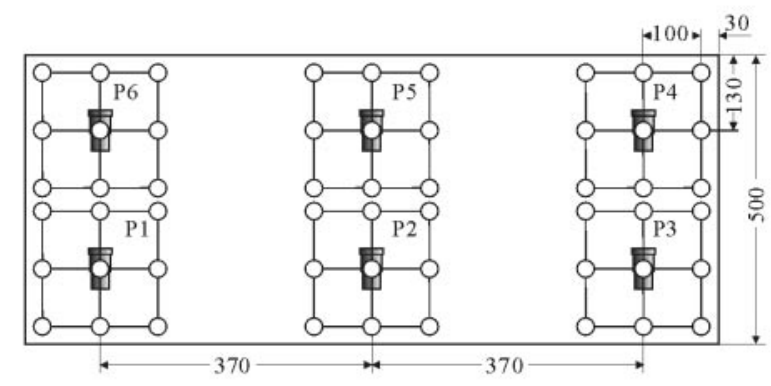

(c)

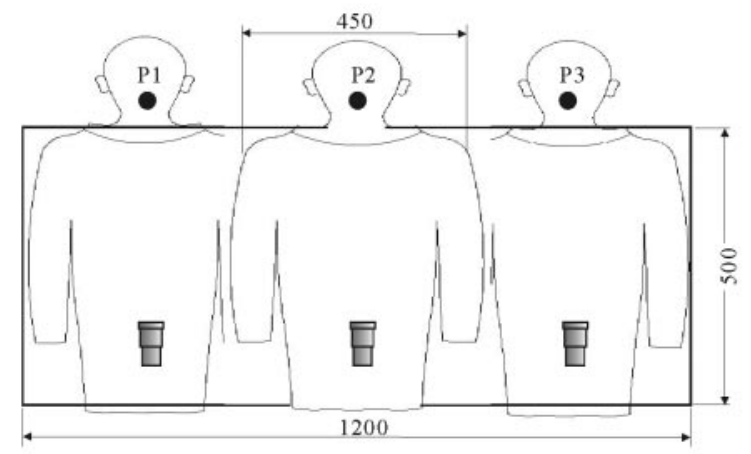

(d)
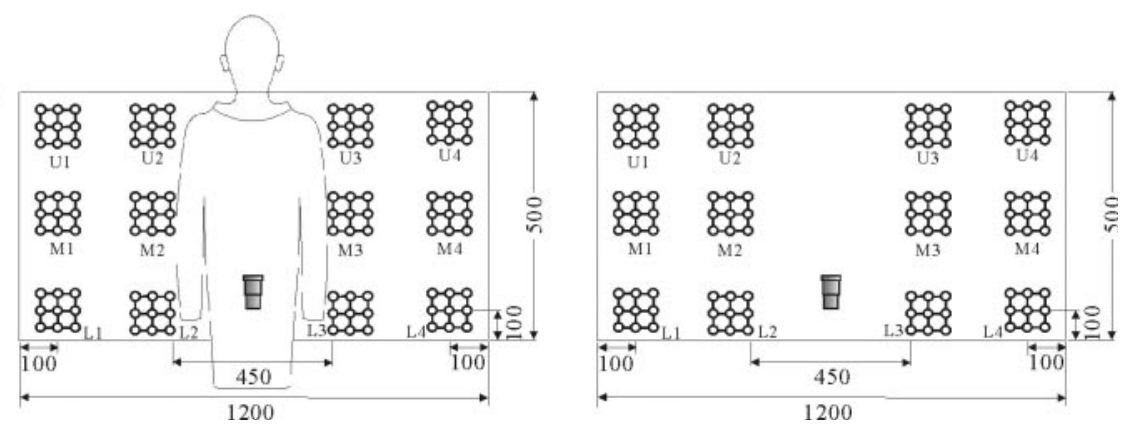

Fig. 3. Sampling grid and source position arrangement by employing the (a) BS 7258:1994 method, (b) prEN 14175:2003 method, (c) ANSI/ASHRAE 110:1995 method, (d) modified method.

above-mentioned nine probes, is positioned in turn at the locations P1 $\sim$ P6 of Fig. 3b when the measurements are performed. The tips of the sampling probes are on the sash plane.

The sampling probes are connected to the sampling manifold by tubes of equal lengths. The detector probe is affixed to the output of the manifold. Tracer gas samples are taken through a stainless steel tube of $8 \mathrm{~mm}$ internal diameter, which is fitted with a diffuser of $30 \mathrm{~mm}$ internal diameter at inlet end of suction velocity $3.7 \mathrm{~cm} \mathrm{~s}^{-1}$. Sampling for the probe-grid positions $\mathrm{P} 1 \sim \mathrm{P} 6$ is taken in turn, $360 \mathrm{~s}$ for each 
grid position, while the tracer gas is released into the cabinet. The data of the initial period of $59 \mathrm{~s}$ are discarded.

Static test following ANSI/ASHRAE 110-1995 standard. The ASHRAE standard uses neat $\mathrm{SF}_{6}$ (the density at 1 bar and $20^{\circ} \mathrm{C}$ is $6.164 \mathrm{~g} \mathrm{l}^{-1}$ ) as the tracer gas, but prEN 14175-3 and BS 7258 reduce the density of the tracer gas close to that of air by using a mixture of $10 \% \mathrm{SF}_{6}$ and $90 \% \mathrm{~N}_{2}$, In general, the emission of high density tracer gas from a local source may cause vertical stratification of the tracer gas concentration (Sandberg and Sjoberg, 1983). In our study, stratification is unlikely to have caused a problem because we used flow visualization to provide details of the aerodynamics and to qualitatively guide the containment experiments. The direction of the momentum of the tracer gas is likely to have a stronger impact on the flow patterns.

The measurements are conducted in accordance with the ANSI/ASHRAE 110-1995 tracer gas containment test method by using $100 \% \mathrm{SF}_{6}$ as the tracer gas. The tracer gas ejector system is manufactured following this test standard. The tracer gas is piped to the ejector, passed through a critical orifice and distributed through a wire mesh outlet diffuser. The flow rate is controlled with a release rate of $41 \mathrm{~min}^{-1}$ by using pressure gauge, a needle valve and a calibrated rotameter. The velocity of the gas at the outlet of the injector is $0.59 \mathrm{~cm} \mathrm{~s}^{-1}$. As shown in Fig. 3c, the center in-line of the ejector is positioned $150 \mathrm{~mm}$ from the horizontal centerline of the sash plane. The manikin with the same shape and size as described previously is fixed at the same position as that in the flow visualization. The ejector and the manikin are placed in three positions: left position, center position and right position. The left and right positions are with the ejector centerline $300 \mathrm{~mm}$ from the inside left wall and the right wall of the cupboard, respectively. The center position is equidistant from the inside walls. Tracer gas samples are taken through a stainless steel tube of $13 \mathrm{~mm}$ internal diameter. The suction velocity is $175.8 \mathrm{~cm} \mathrm{~s}^{-1}$. The detector probe is affixed to the manikin's breathing zone' under the nose of the manikin with the center of the probe $660 \mathrm{~mm}$ above the work surface. The nose of the manikin is $75 \mathrm{~mm}$ in front of the sash plane. The $\mathrm{SF}_{6}$ tracer gas is recorded for a period of $300 \mathrm{~s}$ while the tracer gas is released into the cupboard.

Static test following modified measurement method. This test mode is modified in accordance with the prEN 14175-3:2003 protocol based on the region-by-region measurement. Tracer gas is $10 \%$ $\mathrm{SF}_{6}$ in $\mathrm{N}_{2}$. The tracer gas ejector system is exactly the same as the previous procedure following the ANSI/ASHRAE 110-1995 standard and is arranged with its center in-line equidistant from the inside walls. The release rate of the tracer gas is $41 \mathrm{~min}^{-1}$.
The velocity of the gas at the outlet of the injector is $0.59 \mathrm{~cm} \mathrm{~s}^{-1}$. As shown in Fig. 3d, nine sampling probes are arranged in a grid based on a square area of $100 \mathrm{~mm} \times 100 \mathrm{~mm}$. There are three vertical and three horizontal grid lines separated from each other by $50 \mathrm{~mm}$ in both directions. The central sampling probe grids are positioned on the sash plane with its center probe at points formed by the intersection of three equally spaced lines between the horizontal boundaries of the sash plane with the two outermost lines $100 \mathrm{~mm}$ from the horizontal boundaries. The upper horizontal grid line (containing U1, U2, U3 and U4) is $50 \mathrm{~mm}$ below the upper sash boundary. The lower horizontal grid line (containing L1, L2, L3 and L4) is $50 \mathrm{~mm}$ above the lower sash boundary. The side vertical grid lines (containing U1, M1, L1, U4, M4 and L4, respectively) are $50 \mathrm{~mm}$ from the vertical sash boundaries. The horizontal middle line (containing M1, M2, M3 and M4) is located at the vertical centerline of the sash opening. Tracer gas samples are taken through a stainless steel tube of $13 \mathrm{~mm}$ internal diameter, which is fitted with a diffuser of $30 \mathrm{~mm}$ internal diameter at inlet end of suction velocity $3.7 \mathrm{~cm} \mathrm{~s}^{-1}$. Sampling is taken for $480 \mathrm{~s}$ and the data for the initial period of $180 \mathrm{~s}$ are discarded.

In order to study the effect of the near-wake region of the manikin, measurements are performed for the cupboard with the manikin's presence and the cupboard without the manikin. For the manikin-occupied cupboard, the manikin with the same shape and size as described previously is fixed at the same position as the flow visualization arrangement. The grids (U2, M2, L2) and (U3, M3, L3) are positioned close to the left and right sides, respectively, of the manikin's body. The distance between U2 and U3, M2 and M3 as well as L2 and L3 is $450 \mathrm{~mm}$, which is equal to the manikin's shoulder width. The distance between $\mathrm{U} 2$ and $\mathrm{U} 3$ is $150 \mathrm{~mm}$, which is equal to the manikin's head width. For the unoccupied hood, the test grids are arranged in exactly the same positions as those deployed in the case of the occupied hood.

\section{RESULTS AND DISCUSSION}

\section{Effect of source position and geometric features on flow structure and contaminant leakage}

1 Flow visualization. The visual test provides a clear picture of how the flow characteristics are affected by the source positions. As illustrated in Figs 4 and 5, the smoke released from the right upper and the middle upper level tends to fragment into two routes. One follows the recirculating movement of the upper recirculation zone and the other follows to the rear of the working cupboard. A large proportion of the smoke is entrained into the upper 

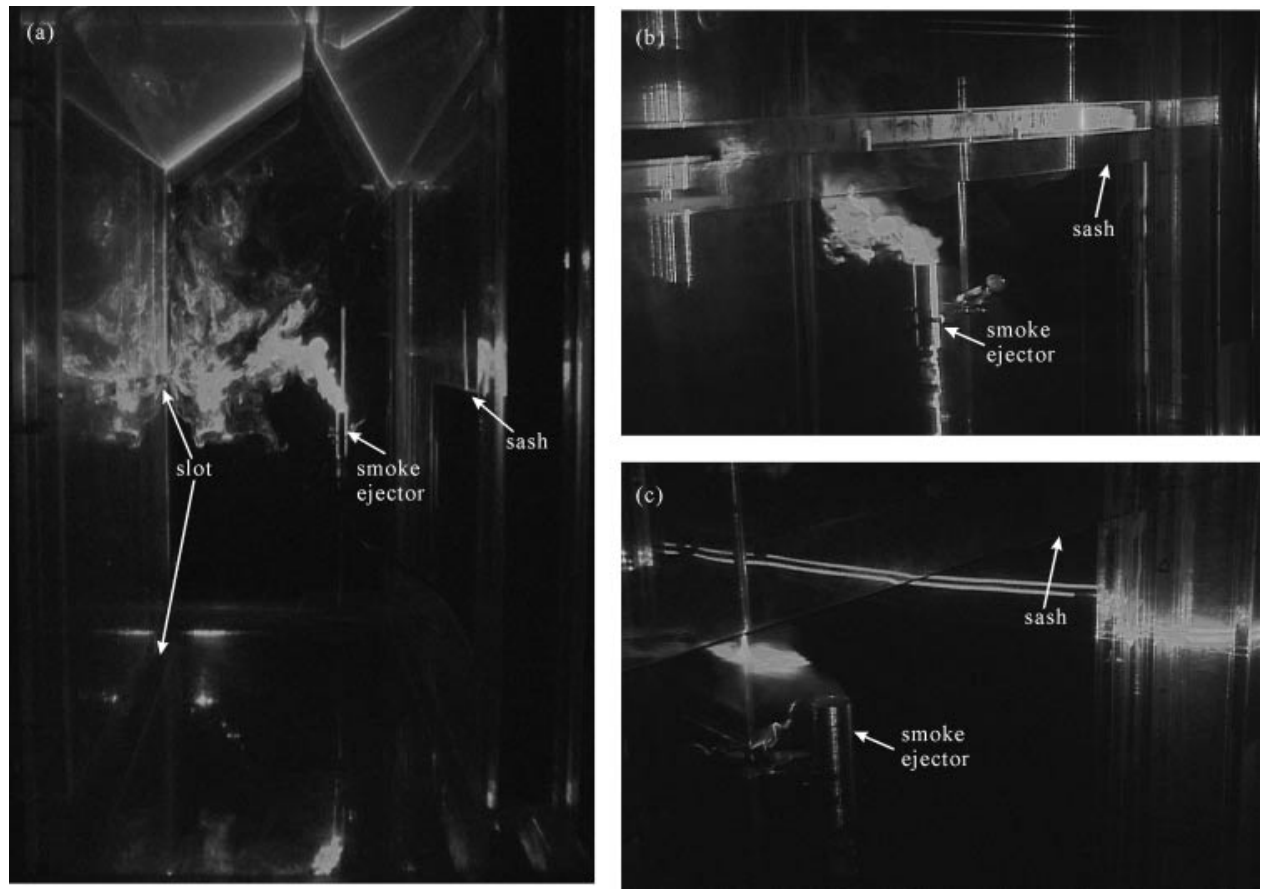

Fig. 4. Side view of smoke patterns in interior of cupboard as source released from right upper region. (a) inside the cupboard, (b) horizontal plane, (c) vertical plane. Sash height $=50 \mathrm{~cm}$.
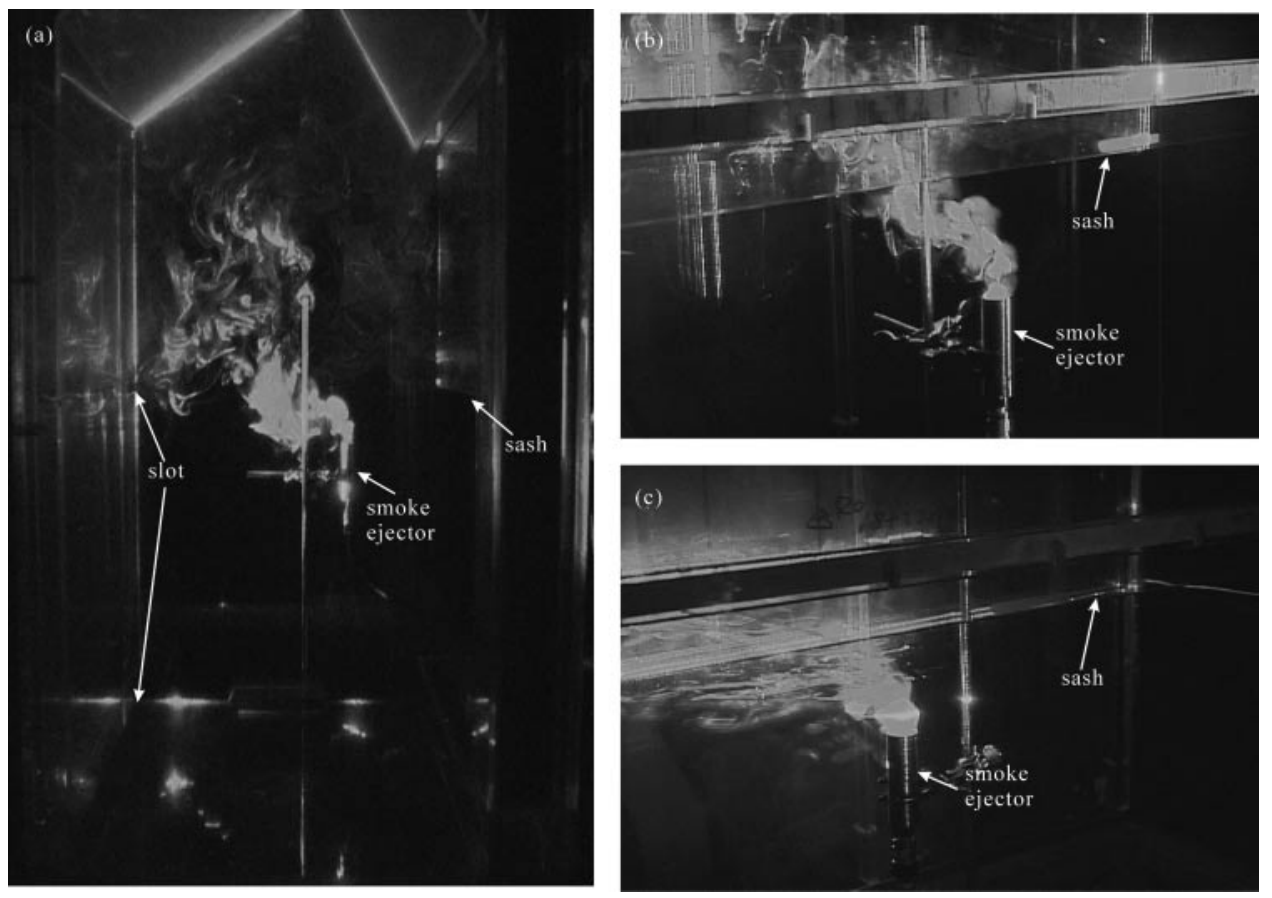

Fig. 5. Side view of smoke patterns in interior of cupboard as source released from middle upper region. (a) inside cupboard, (b) horizontal plane, (c) vertical plane. Sash height $=50 \mathrm{~cm}$.

recirculation zone behind the sash. Unsteady, complex three-dimensional vortices can be observed there. A number of vortices are generated continuously and stretched downward due to the strong suction of the exhaust system. The inflowing stream has little or no turbulence as it passes through the face of the cupboard. The shear layer at the boundary of the upper edge rolls up in a more laminar manner 
before becoming turbulent. The transport of the vortices upstream, where they catch up and merge with previously shed vortices along the upper region of the chamber, yields a much faster and more uniform flow through the face of the cupboard. These vortices persist over time, smoothly propagated along side the main stream. It is postulated that the flow released from the upper level allows very little contaminant escape from the chamber.

However, the development of flow from the lower level source exhibits a quite different pattern. The unsteady three-dimensional recirculation zones are produced near the right bottom corner and the middle bottom wall, as illustrated in Figs 6 and 7 . The findings suggest that these recirculation zones are the result of turbulent flow separation. The boundary layer of the flow separates from the surface of bottom wall of the cupboard forms a shear layer and is highly unstable. Due to the complex interaction between existing vortices and the surrounding wall, a number of vortices are generated. They appear to evolve from the complex dynamics of the complex three-dimensional turbulent flow. In turbulent flow the air moves irregularly causing continuous exchange of momentum and the momentum exchange is the reason for the turbulent shear stress. Because of the existence of the three-dimensional flow and the recirculation zones induced near the bottom wall, the contaminant will inevitably be carried to the recirculation zone. The contaminant leakage from these areas would thus be very possible because of the significant turbulent dispersion occurring there.

(2) Tracer gas performance verification.. In the present study, the British method and the European proposed standard were conducted: I. BS 7258: part 4:1994 standard and II. prEN 14175-3:2003 protocol.

I. Results obtained by employing the BS 7258: part 4:1994 method: The measured $\mathrm{SF}_{6}$ concentrations, according to the BS 7258: part 4:1994 method, are the averages over the entire multiple grids along the cupboard face. Figure 8 shows the values of $\mathrm{SF}_{6}$ leakage for the source released from the upper level (S4, S5 and S6 in Fig. 3a) are below 0.09 ppm. However, the values of $\mathrm{SF}_{6}$ concentration increase as much as a 100-fold when the source is released from the lower level, i.e. the positions S1, S2 and S3. The tracer gas test results support the evidence of the visual findings. As stated in the previous discussion, the flow consists of an upper uniform regime and the bottom-boundary recirculation regime close to the side walls and the doorsill, due to the inherent flow physics. For the upper level contaminant source, the smoke that is not entrained into the vortex is carried smoothly to the cupboard. On the other side, the bottom-boundary recirculation regime where the separation of the flow takes place contributes to the serious contaminant leakage. The severe three-dimensional recirculation patterns occurring at the side corners and the doorsill regions due to the lower level contaminant source would
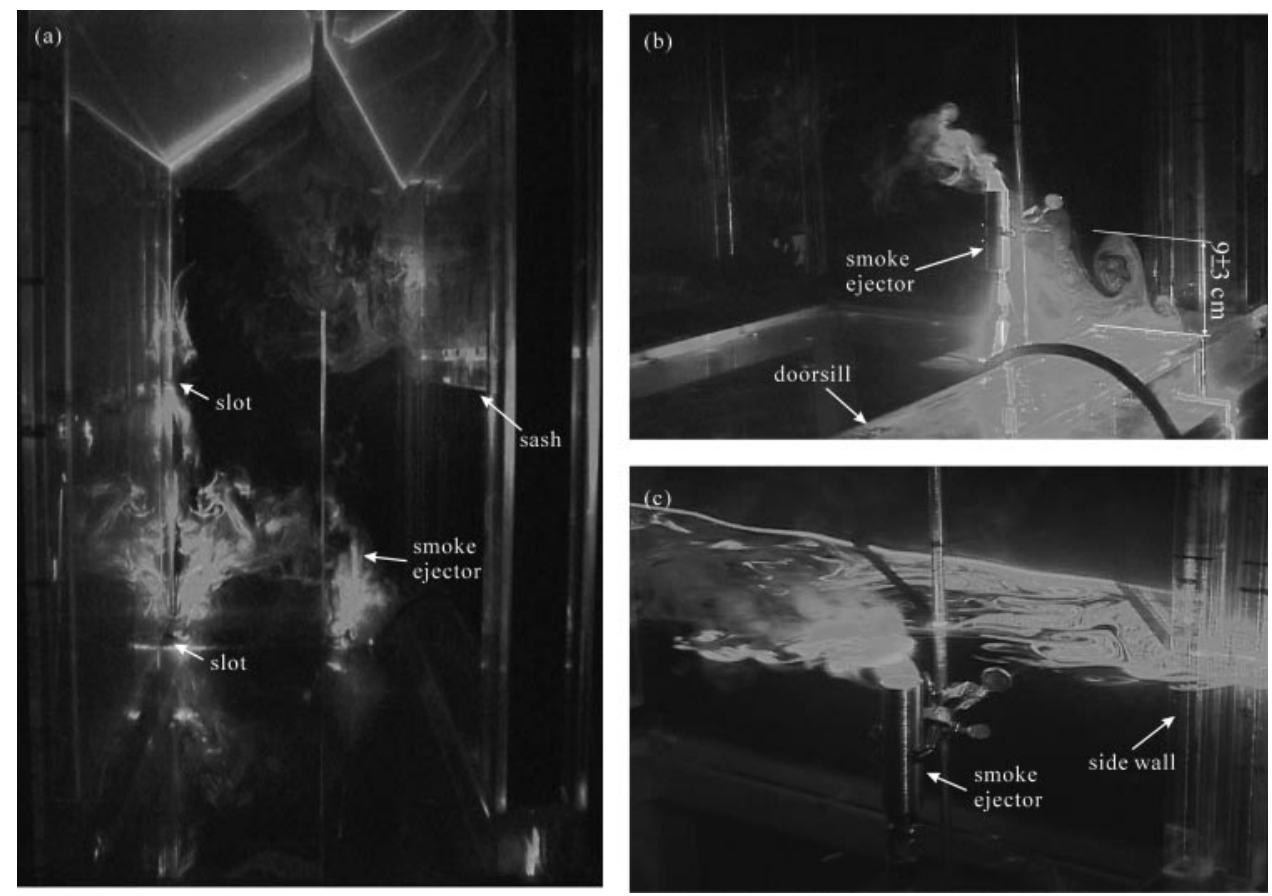

Fig. 6. Side view of smoke patterns in interior of cupboard as source released from right bottom corner. (a) inside cupboard, (b) horizontal plane, (c) vertical plane. Sash height $=50 \mathrm{~cm}$. 

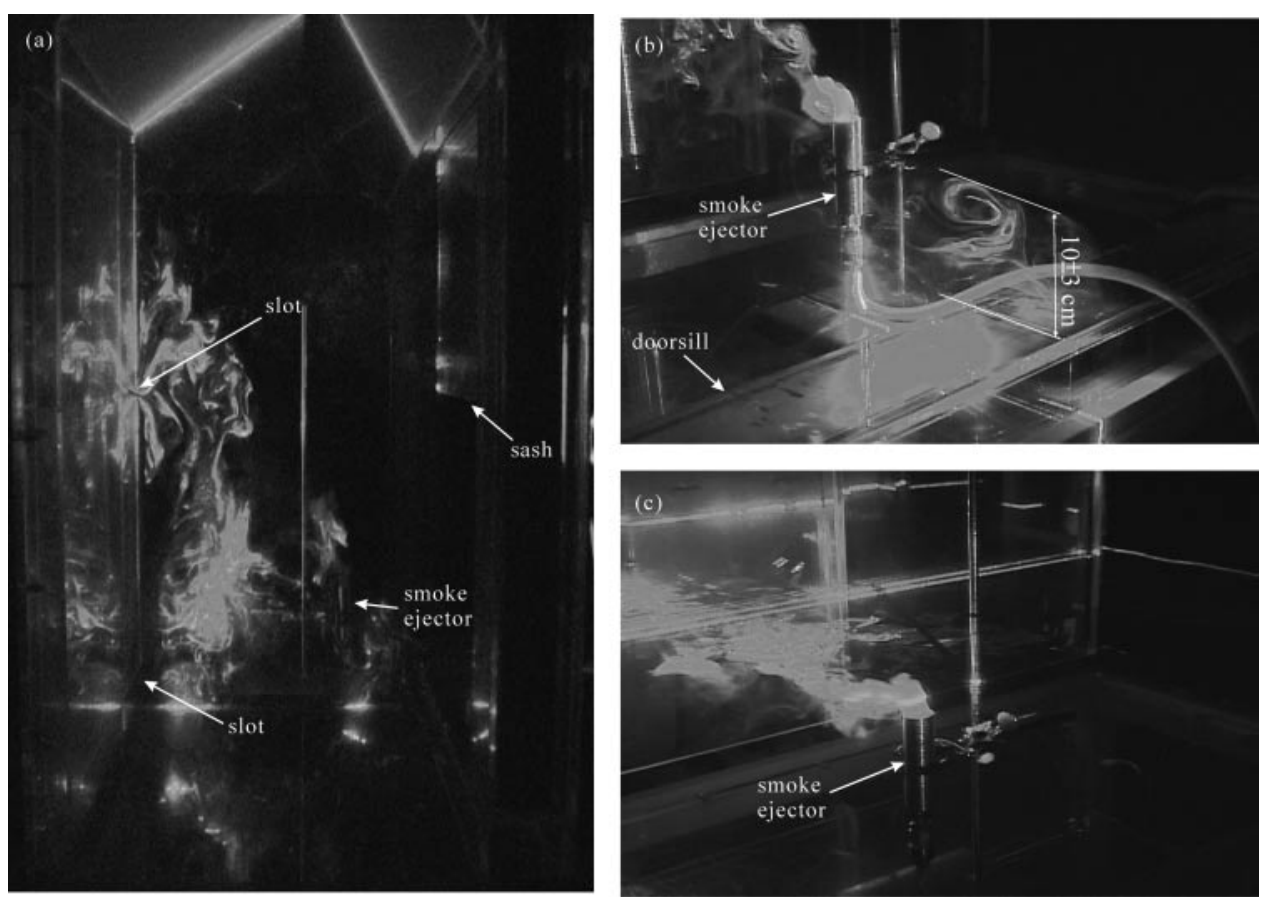

Fig. 7. Side view of smoke patterns in interior of the cupboard as source released from middle bottom region. (a) inside cupboard, (b) horizontal plane, (c) vertical plane. Sash height $=50 \mathrm{~cm}$.

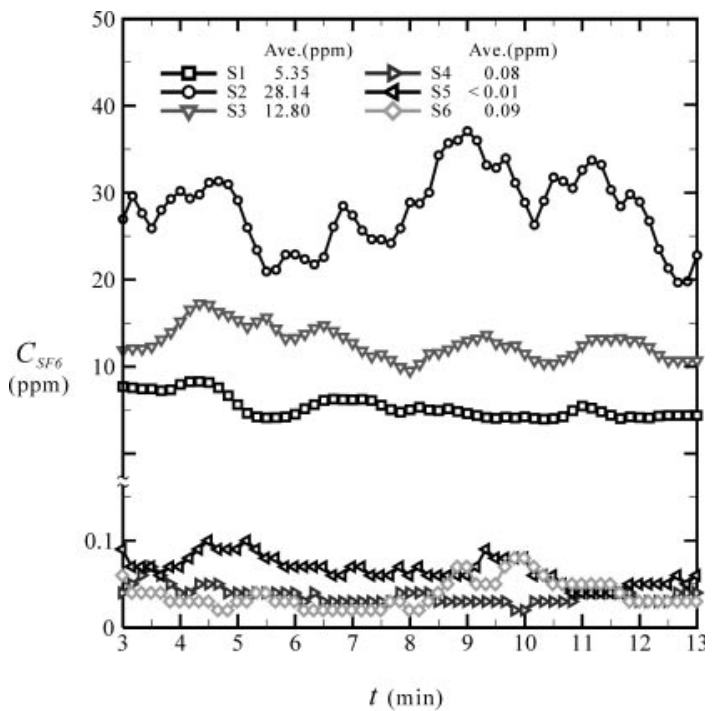

Fig. 8. Time histograms of $\mathrm{SF}_{6}$ leakages by employing BS 7258: part4:1994 tracer gas test method.

be responsible for the spread of the contaminant leakages.

The simultaneous sampling strategy described in this test method may only reflect the concentration of the bulk samples across multiple locations through the cupboard. This may hint that the cleaner' samples released from the upper uniform regime will mix and dilute the more contaminated' samples taken from the boundary recirculation regime. The problem for measuring samples collected here may show a lack of

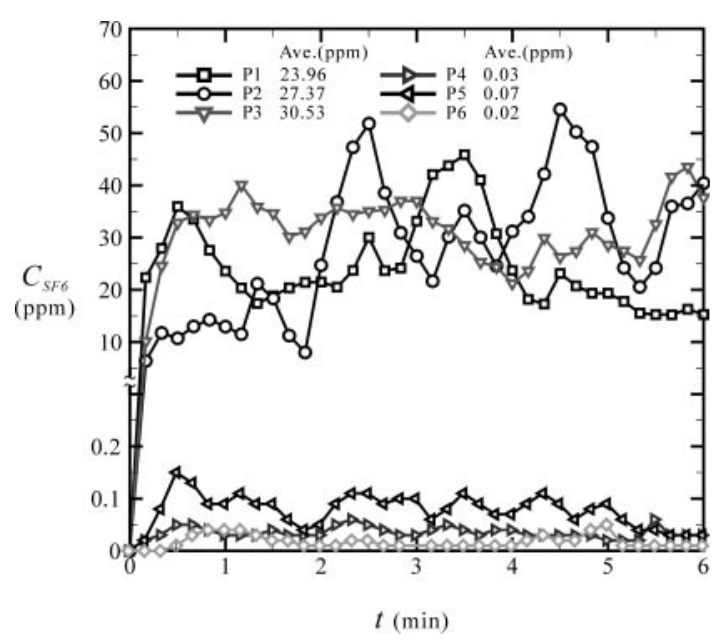

Fig. 9. Time histograms of $\mathrm{SF}_{6}$ leakages by employing prEN 14175-3:2003 tracer gas test method.

variation because each individual region of the cupboard may cause a large spatial variation in aerodynamic features. This situation is particularly important since the release of highly toxic substances where high impacts on a short time scale may be extremely dangerous.

II. Results obtained by employing prEN 141753:2003 protocol: The underlying feature in the prEN 14175-3:2003 methodology is to detect the time-weighted mean concentration of $\mathrm{SF}_{6}$ leakage within each individual localized area of the cupboard face, as shown in Fig. 3b. The tracer gas results 

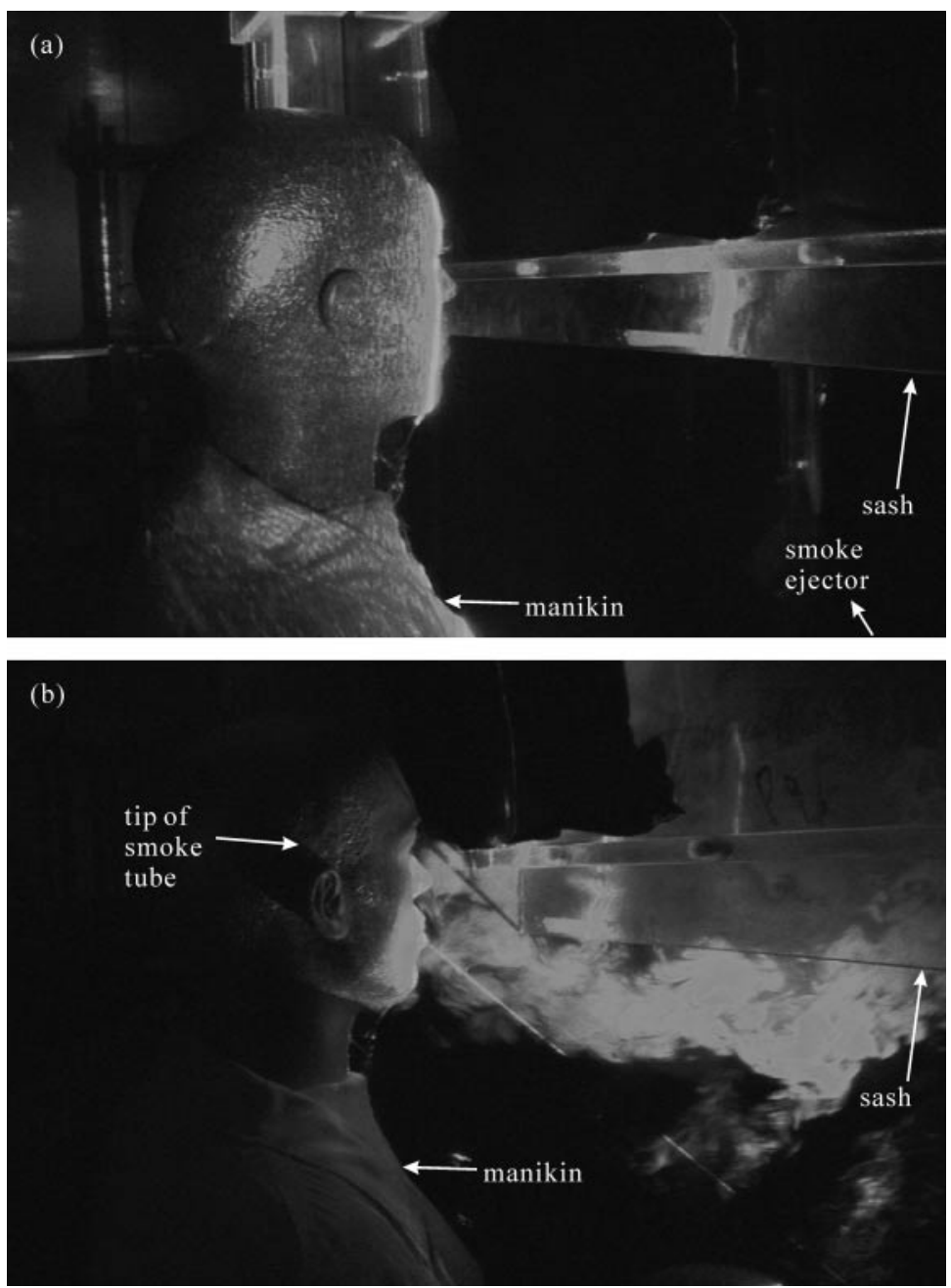

Fig. 10. Smoke patterns around breathing zone of manikin. Smoke released from (a) smoke ejector and (b) head side of the manikin.

confirm that it would be logical to choose the source height as well as source position as important factors of the cupboard performance. The levels of $\mathrm{SF}_{6}$ leakage measured at the upper locations, the grids $\mathrm{P} 4$, $\mathrm{P} 5$ and P6 of Fig. 3b, are quite low, as shown in Fig. 9. However, substantially higher $\mathrm{SF}_{6}$ concentrations are detected at the grids $\mathrm{P} 1, \mathrm{P} 2$ and $\mathrm{P} 3$, i.e. at the bottom edge of the cupboard.

As discussed above, the recirculation flow structures contribute to the serious contaminant leakage, particularly in the regions near the doorsill and the lower level side poles. From Figs $6 \mathrm{~b}$ and $7 \mathrm{~b}$, it is noted that the height of the recirculation zone at the edge of the cupboard is $\sim 6-13 \mathrm{~cm}$. The recirculation zones consist of a large quantity of entrained $\mathrm{SF}_{6}$. Because the central test grids, in accordance with this method, are positioned at a distance of $13 \mathrm{~cm}$ from the edge of the side corners and the doorsill, the arrangement of this test protocol is likely to be able to detect high $\mathrm{SF}_{6}$ concentration. The large-scale vortex structures occurring near the perimeter edge concur with the high degree of contaminant leakage in those areas. The evolution of the concentration fluctuations, showing the multi-peak pattern of $\mathrm{SF}_{6}$ concentration, is strongly affected by the turbulent dispersion across the separated boundary layers near the walls and served to reveal the essential features of the contaminant transport process. We conclude that the methodology of the prEN 14175-3:2003 protocol produces more reliable and consistent results based on the region-by-region measurement, encompasses the most area of the entire recirculation zone of the cupboard and predicts the maximum $\mathrm{SF}_{6}$ concentration contour level within each individual area of the cupboard.

\section{Effect of manikin presence on flow structure and contaminant leakage}

The effect of the wake on worker exposure has been studied experimentally by Kim and Flynn (1992) and 
Flynn and Ljungqvist (1995). One deficit of the two test methods described previously is the failure of considering contaminant leakage with respect to the wake region of the operator's body. In order to study the effect of the near-wake region of the manikin, measurements are performed as follows:

\section{(1) Flow visualization.}

I. Flow patterns at breathing zone of manikin: When the smoke is released from the smoke ejector, no trace of smoke is found in the breathing zone of the manikin, as shown in Fig. 10a. As the smoke is introduced upstream of the breathing zone of the manikin to visualize the flow patterns, as shown in Fig. 10b, the smoke is directed into the fume chamber without any reversal. This type of flow will push contaminants into the hood and make them very difficult to escape.

II. Flow patterns around chest of manikin: When the smoke is released from the smoke ejector, no smoke is found in front of the manikin's chest, as shown in Fig. 11a. However, as the smoke is introduced into the upstream of the manikin's chest shown in Fig. 11b, the flow exhibits a large region of separated reverse flow in front of the chest. The recirculation region may transport the vortices downstream where they catch up and merge with previously shed vortices along the region of the manikin's chest. Contaminants may then be transported into the breathing zone of a worker or other areas from sources located within the reverse flow region. The chest recirculation is still a dangerous flow structure that may cause contaminant leakage. This should be taken into consideration when fume hoods are designed.

III. Flow patterns around hip of manikin: Highly unstable three-dimensional recirculation zones are observed in the horizontal and vertical planes around the side corner and doorsill area, as shown in Figs 12a and $b$, respectively. A number of continuously generated large rotating vortices are induced at these areas. Large vortices persist and propagate along the stream until they reach fairly far downstream. The recirculation zone induced by the flow separation
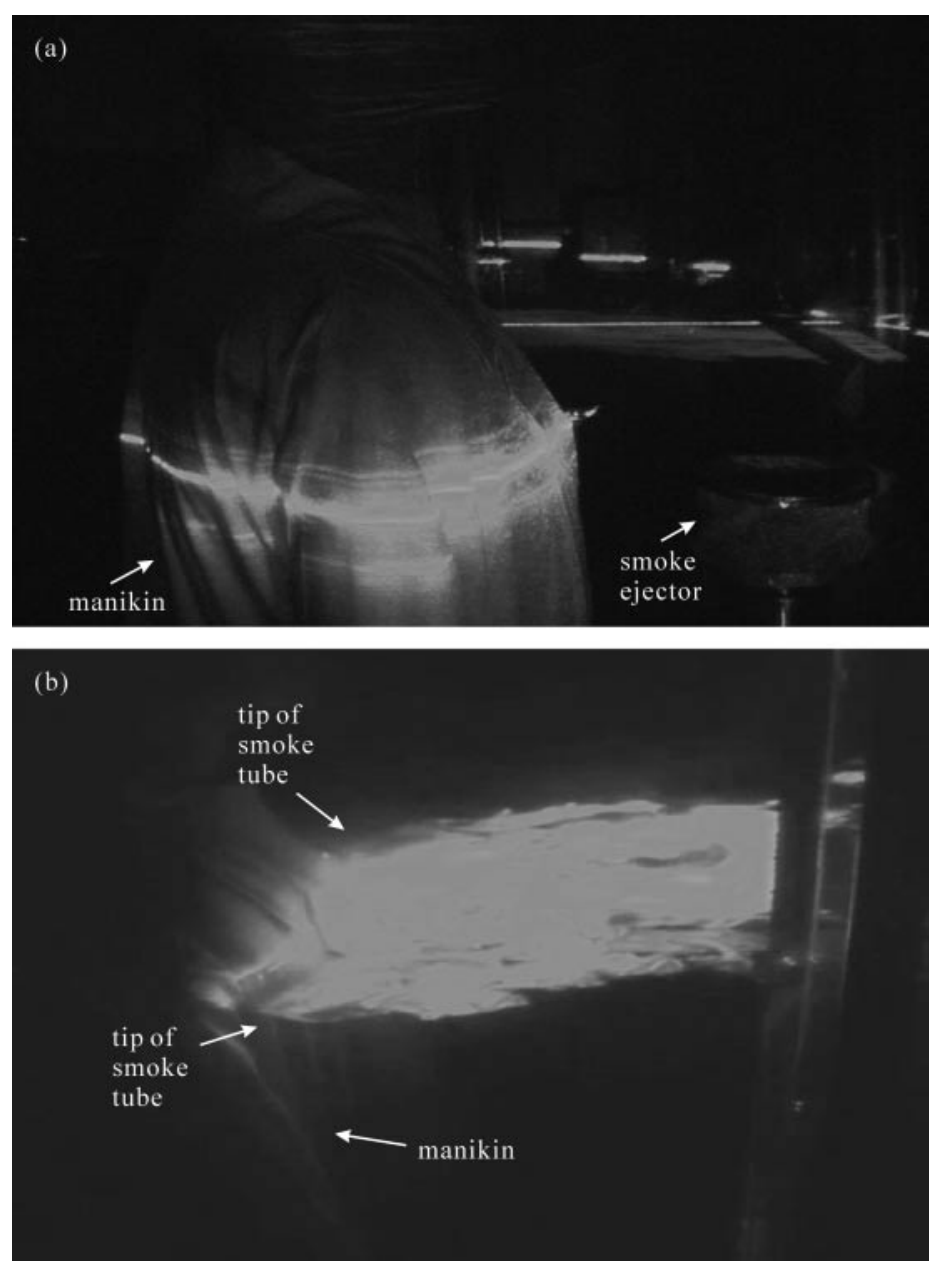

Fig. 11. Smoke patterns in front of chest of manikin. Smoke released from (a) smoke ejector and (b) chest side of manikin. 

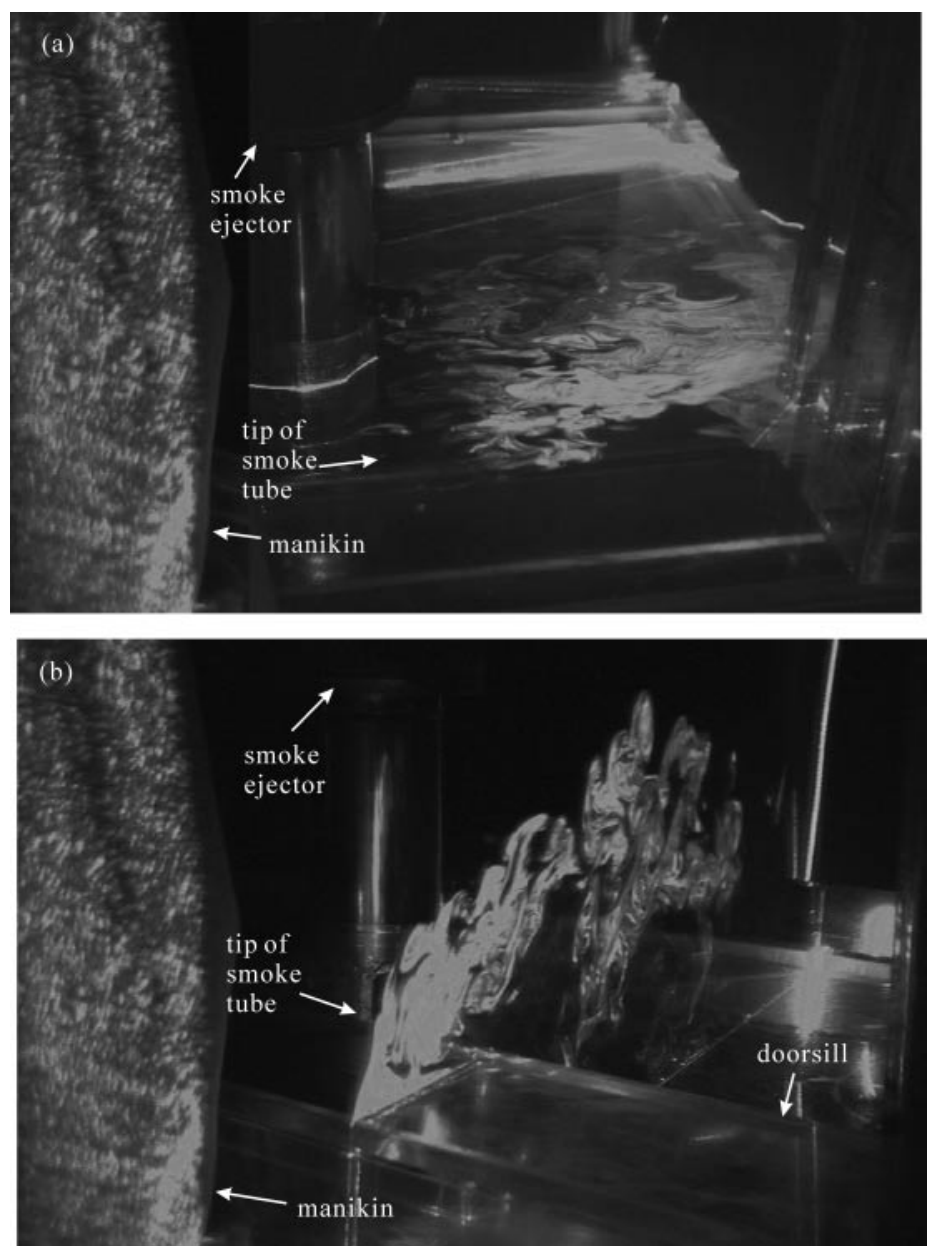

Fig. 12. Smoke patterns around doorsill as manikin placed in front of hood. Smoke released from (a) smoke ejector and (b) middle bottom wall.

around the near-wake region of the manikin shown in Fig. 12 is apparently larger than that without manikin's presence shown in Figs 13a and b. The presence of the manikin creates a wake zone accompanied by vortex formation and have the effect of enlarging recirculation than its counterpart of the manikin unoccupied case.

\section{(2) Tracer gas performance verification.}

I. Results obtained by employing ANSI/ASHRAE 110-1995 method: The principle of the ANSI/ ASHRAE 110-1995 method is to measure $\mathrm{SF}_{6}$ concentration in the breathing zone of a fume cupboard operator, as shown in Fig. 3c. Figure 14 shows that the measured time-weighted mean concentrations of $\mathrm{SF}_{6}$ at the breathing zone of the manikin are nearly undetectable at the left (P1), center (P2) and right (P3) positions. The result confirms the visual flow patterns of Fig. 10a that the air around the breathing zone' goes directly into the hood so that backward dispersion is limited.
The sampling probe in accordance with this standard is positioned in the breathing zone of the manikin and $75 \mathrm{~mm}$ from the face of the cupboard. Because the test zone is not adjacent to the side walls and the doorsill of the cupboard, the contaminant dispersion from these recirculation areas may not give appreciable concentrations in the test zone. Traditionally, it is common in the field of occupational hygiene to evaluate worker's exposure to contaminant leakage by measuring their concentration in the breathing zone. However, such samples may not detect serious leakages from other parts of the cupboard opening, which may be unacceptable for highly toxic materials.

II. The performance verification of the modified containment test method: A modified test method is proposed to offer a greater clarification of the behavior of the flow structure within the near-wake of the manikin and the contaminant leakage. The modified test mode combined with the region-byregion approach similar to the prEN 14175-3 proto$\mathrm{col}$, and the measurements within the near-wake of 

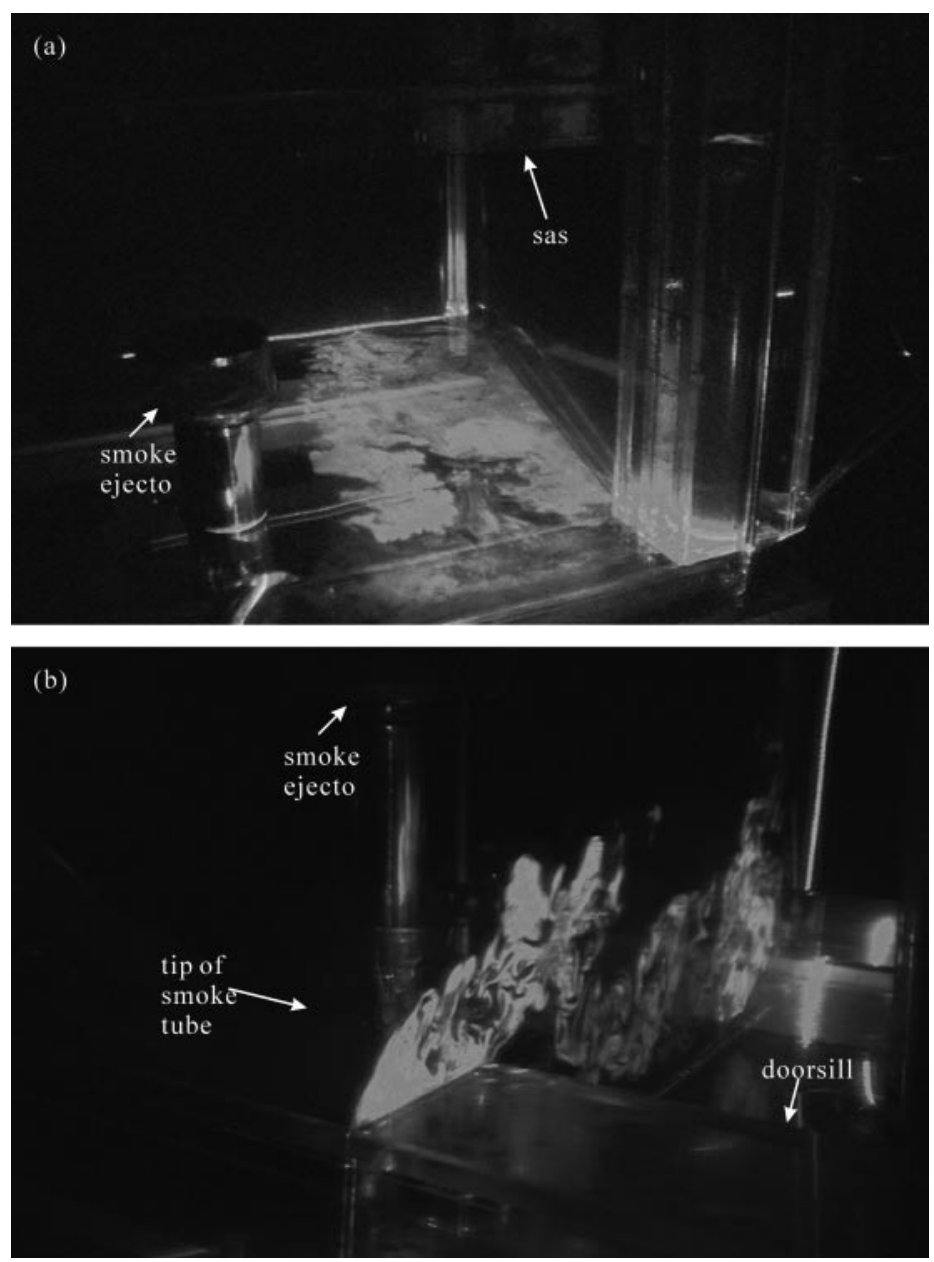

Fig. 13. Smoke patterns around doorsill as manikin placed in front of the hood. Smoke released from (a) smoke ejector and (b) middle bottom wall.

the manikin, show substantially different results for the containment ability. Table 1 shows that the levels of $\mathrm{SF}_{6}$ leakages measured at the upper grids, i.e. the grids U1, U2, U3 and U4, are quite low for the cupboard without manikin's presence. However substantially high $\mathrm{SF}_{6}$ concentrations are detected at the grids M1, M4, L1, L2, L3 and L4, i.e. at the middle and bottom side edge of the cupboard. The tracer gas and visual findings are in good qualitative agreement, showing that the large-scale vortex structures occur near the side edges of the cupboard may induce strong turbulence and therefore enhance dispersion of contaminant.

The presence of the manikin is likely to have a significant impact on the magnitude of the contaminant leakages. Table 1 demonstrates the levels of $\mathrm{SF}_{6}$ leakages around the breathing zone of the manikin, i.e. grids U2 and U3, are quite low. The downward flushing current may cause the deviation from the top of the manikin head and would not induce the recirculation zone here. The values of $\mathrm{SF}_{6}$ concentration around the sides of the chest, i.e. grids M2 and M3, are nearly undetectable. The chest recirculation does not seem to entrain the $\mathrm{SF}_{6}$ out of the cupboard. However, the values of $\mathrm{SF}_{6}$ leakage from the cupboard with manikin's presence, which are measured around the near-wake region near the bottom wall (at the manikin's hip level), i.e. positions L2 and L3, are nearly 2-4 times those without the manikin's presence. Around the corner region, i.e. positions L1 and L4, they are nearly 17-22 times higher than those of the cupboard without the manikin's presence. It is known that highly unstable recirculation zones are observed along the side corner and the doorsill, while the manikin is placed in front of the cupboard. Therefore, contaminant leakages may occur here, and also in the region near the operator. In the presence of the turbulent wake produced by the manikin, the reverse flow eddies form and subsequently shed downstream are important in governing the transport of containment in these regions. The wake zone disrupts and draws contaminant from a vortex where leakage may build up at the hood face and extend into the neighboring region. An effect upon both the 


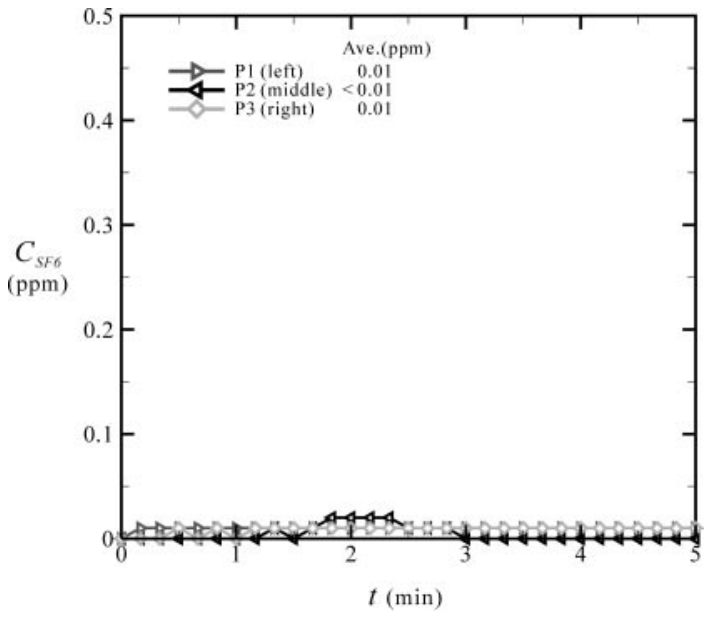

Fig. 14. Time histograms of $\mathrm{SF}_{6}$ leakages by employing ANSI/ASHRAE 110:1995 tracer gas test method.

Table 1. $\mathrm{SF}_{6}$ concentrations $\boldsymbol{C}_{\boldsymbol{S F \boldsymbol { G }}}$ measured by employing the modified method

\begin{tabular}{lcc}
\hline Grid position & $\begin{array}{l}\mathrm{C}_{\mathrm{SF6}} \text { with manikin } \\
\text { mean (peak), ppm }\end{array}$ & $\begin{array}{l}\mathrm{C}_{\mathrm{SF6}} \text { without manikin } \\
\text { mean }(\text { peak), ppm }\end{array}$ \\
\hline $\mathrm{U} 1$ & $0.25(0.37)$ & $0.22(0.54)$ \\
$\mathrm{U} 2$ & $0.01(0.04)$ & $0.01(0.02)$ \\
$\mathrm{U} 3$ & $0.06(0.09)$ & $<0.01(0.01)$ \\
$\mathrm{U} 4$ & $0.65(1.22)$ & $0.12(0.32)$ \\
$\mathrm{M} 1$ & $3.84(5.33)$ & $0.71(3.01)$ \\
$\mathrm{M} 2$ & $<0.01(0.01)$ & $<0.01(0.01)$ \\
$\mathrm{M} 3$ & $<0.01(0.01)$ & $<0.01(0.01)$ \\
$\mathrm{M} 4$ & $5.28(8.59)$ & $0.76(2.80)$ \\
L1 & $8.24(30.20)$ & $0.38(2.28)$ \\
L2 & $5.33(11.94)$ & $1.20(3.11)$ \\
L3 & $1.28(2.51)$ & $0.64(2.87)$ \\
L4 & $4.96(7.70)$ & $0.30(0.70)$ \\
\hline
\end{tabular}

complex interaction between the two shear layers separated from each side of the manikin and the doorsill of the cupboard subsequently cause more contaminant leakages. The test mode proposed is useful to verify the correlation between the detailed flow patterns and the contaminant transport behavior. From the experimental results, it is convinced that the effect of the geometry feature of the cupboard is of great importance in contaminant leakage, especially in the vicinity of the near-wake of the operator.

\section{CONCLUSIONS AND RECOMMENDATIONS}

The overall concentration trend of contaminants along the face of the fume cupboard is obtained experimentally. Flow visualization tests demonstrate detailed flow patterns in different regions of the cupboard to qualitatively support the experimental results of the tracer gas measurements. The following conclusions are drawn from the results:

1. The airflow patterns and performance of the laboratory fume cupboard are closely correlated. The source position, geometric features and presence of an operator are important to the laboratory fume hood performance. Large-scale vortex structures are found to exist around the side walls and the doorsill of the cupboard and in the vicinity of the near-wake region of the operator. These areas with drastic flow recirculation are corresponding to the regions where significant high contaminant leakages are detected.

2. A comprehensive strategy for detection of the cupboard leakage should base on the combination of region-by-region approach and the measurement of the near-wake of an operator. It is obvious that the test data derived from the ANSI/ASHRAE 110-1995 method (sampling at the breathing zone) for measuring cupboard performance are inadequate for describing the effectiveness of the cupboard. When good containment results are obtained by employing this standard, the problem may rise from the lack of understanding the flow details, and this is a topic of extreme importance in the correct interpretation of the test result of good' performance.

3. The purpose of the present study was to find a relationship between the static' test and exposure during the fume cupboard operation. However, the performance of the cupboard is strongly influenced by the ventilation system and other features of the laboratory in which it is installed. We suggest that a better understanding of the roles of the dynamic' factors, such as rapidly sash opening and people walk-by, in the fume cupboard performance is clearly an area for further investigation.

\section{NOMENCLATURE}

$C_{S F 6}=$ concentration of $\mathrm{SF}_{6}$, in ppm

$S=$ position of smoke ejector according to BS 7258:1994

$U=$ upper horizontal sampling grid according to modified method

$M=$ middle horizontal sampling grid according to modified method

$L=$ lower horizontal sampling grid according to modified method

$P=$ sampling grid according to ANSI/ASHRAE 110:1995 and prEN 14175-3:2003 method

$X=$ horizontal coordinate originated at left side boundary of hood opening in sash plane

$Y=$ vertical coordinate originated at left side boundary of hood opening in sash plane

$Z=$ normal coordinate originated at left side boundary of hood opening in sash plane $t$ sampling time 
Acknowledgements-This research is supported by the Minister of Education of Taiwan. R.O.C. under grant number 0940081791.

\section{REFERENCES}

American Society of Heating, Refrigeration and Air Conditioning Engineers (ASHRAE). (1995) Method of testing performance of laboratory fume hoods (ANSI/ASHRAE Standard 110-1995). Atlanta, GA: ASHRAE.

British Standards Institution (BSI). (1994) Laboratory fume cupboards. Part 4. Method for determination of the containment value of a laboratory fume cupboard (BS 7258: Part 4: 1994). London: BSI.

Durst F, Pereira JC. (1991) Experimental and numerical investigations of the performance of fume cupboards. Build Environ; 59: 153-64.

Durst F, Pereira JCF. (1992) Numerical simulation and optimization of the aerodynamics flow field inside fume cupboards. J Wind Eng Indl Aerodyn; 40: 127-45.

Ekberg LE, Melin J. (2000) Required response time for variable air volume fume hood controllers. Ann Occup Hyg; 44: 143-50.

European Committee for Standardization (CEN). (2003) Fume Cupboards-Parts 3: Type test methods (prEN 14175-3). CEN, Brussels.

Flagan RC, Seinfeld JH. (1988) Fundamentals of air pollution engineering. NJ, USA: Prentice Hall, Englewood Cliffs, pp. 290-357.

Fletcher B, Johnson AE. (1992a) Containment testing of fume cupboards-I, methods. Ann Occup Hyg; 36: 239-52.

Fletcher B, Johnson AE. (1992b) Containment testing of fume cupboards-II, test room measurements. Ann Occup Hyg; 36:395-405.

Flynn MR, Ljungqvist B. (1995) A review of wake effects on worker exposure. Ann Occup Hyg; 39: 211-21.

Hu P, Ingham DB, Wen X. (1996) Effect of location of the exhaust duct, an exterior obstruction and handle on the air flow inside and around a fume cupboard. Ann Occup Hyg; 40: 127-44.

Hu P, Ingham DB, Wen X. (1998) Effect of baffles and a louvered bypass on the airflow and the convective patterns of contaminant inside a fume hood. Am Ind Hyg Assoc J; 59: $303-12$.

Johnson AE, Fletcher B, Saunders CJ. (1996) Air movement around a worker in a low-speed flow field. Ann Occup Hyg; 40: 57-64.

Kim T, Flynn MR. (1992) The effect of contaminant source momentum on a worker's breathing zone concentration in a uniform freestream. Am Ind Hyg Assoc J; 53: 757-66.

Kirkpatrick AT, Reither R. (1998) Numerical simulation of laboratory fume hood airflow performance. Career and Technical Education, ASHRAE Transactions: Symposia; 104: 999-1011.

Lan NS, Viswanathan S. (2001) Numerical simulation of airflow around a variable volume/constant face velocity fume cupboard. Am Ind Hyg Assoc J; 62: 303-12.

Marzal F, Gonzalez E, Minana A et al. (2002) Determination and interpretation of total and transversal linear efficiencies in push-pull ventilation systems for open surface tanks. Ann Occup Hyg; 46: 629-35.

Marzal F, Gonzalez E, Minana A et al. (2003a) Methodologies for determining capture efficiencies in surface treatment tanks. Am Ind Hyg Assoc J; 64: 604-8.

Marzal F, Gonzalez E, Minana A et al. (2003b) Visualization of airflows in push-pull ventilation systems applied to surface treatment tanks. Am Ind Hyg Assoc J; 64: 455-60.

Maupins K, Hitchings DT. (1998) Reducing employee exposure potential using the ANSI/ASHRAE 110 Method of Testing Performance of Laboratory Fume Hoods as a diagnostic tool. Am Ind Hyg Assoc J; 59: 133-8.

Nicholson GP, Clark RP, Mervyn L. (2000) Computational fluid dynamics as a method for assessing fume cupboard performance. Ann Occup Hyg; 44: 203-17.

Sandberg M, Sjoberg M. (1983) The use of moments for assessing air quality in ventilated rooms. Build Environ; 18: 181-97.

Saunders T. (1993) Laboratory fume hoods, a user's manual. New York: John Wiley \& Sons, Inc, Professional, Reference and Trade Group, pp. 56.

Volin CE, Joao RV, Reiman JS et al. (1998) Fume hood performance: face velocity variability inconsistent air volume systems. Appl Occup Environ Hyg; 13: 656-62. 
